\title{
La psychopathologie antique: sources, historiographie et perspectives
}

Gwenaëlle Le Person

Keywords: madness in antiquity; antiquity historiography; soul; medicine; philosophy; literature; archaeology

Il peut paraître étonnant que, dans le sillage des écrits de Michel Foucault ${ }^{1}$, aucune histoire de la folie dans l'Antiquité gréco-romaine n'ait été publiée. Cela tient vraisemblablement à deux choses. A l'instar de la notion occidentale contemporaine de folie qui est multiple, polymorphe et ne présente que des sens fracassés ${ }^{2}$ tant sa délimitation est imprécise, elle est, dans le monde gréco-romain, diffractée dans de nombreux genres de discours et n'apparaît qu'en contre-jour de savoirs et de cultures déterminés. De fait, ainsi que l'estime Israel E. Drabkin dans son article programme pour une étude sur la psychopathologie antique, si faire une histoire de la folie antique apparaît comme un sujet d'étude passionnant, il faudrait, pour y parvenir, associer des spécialistes d'horizons différents tant la «biologie, la psychologie, la philosophie, la médecine, la magie, la religion, la loi, l'art, la littérature, tous ces territoires ont un réel et intime contact avec le sujet de l'esprit et ses aberrations $»^{3}$.

1 Foucault 1961.

2 Foucault 1961, 181.

3 Drabkin 1955, 223.

Gwenaëlle Le Person, Université de Rennes 2, LAHM, UMR 6566, Place du recteur H. Le Moal, F-35000 Rennes (tresmeur@club-internet.fr). 
Ecrire une histoire de la folie antique apparaît donc comme un projet complexe et ambitieux, ce qui ne signifie pas pour autant qu'aucune recherche n'ait été menée sur ce sujet. La psychopathologie antique est un champ historiographique relativement récent qui s'est progressivement structuré et étoffé. Le rappel des étapes de sa structuration permet de dresser un état des lieux des enquêtes effectuées et des perspectives de recherche.

\section{La naissance de la science psychiatrique et le nouveau regard porté sur la folie antique}

Le traité médico-philosophique de Philippe Pinel, sur la manie, paru en $1801^{4}$, témoigne de l'importance de l'héritage antique dans la conception et la pratique de la médecine du XIX ${ }^{\mathrm{e}}$ siècle. A cette période encore, les théories médicales antiques ne sont pas étudiées pour elles-mêmes mais constituent un support de référence pour la science psychiatrique naissante, qui demeure largement empirique. Avec la mise en place d'une réglementation psychiatrique dès 1838 à l'instigation de Jean-Dominique-Etienne Esquirol, la création d'une étiologie psychique des maladies mentales par Jean-Martin Charcot puis la naissance de la psychanalyse freudienne, la psychopathologie antique commence à devenir un champ historiographique à part entière. La structuration de ce dernier pâtit cependant, à ses débuts, d'un manque de distance des chercheurs avec la science psychiatrique et psychanalytique contemporaine qu'ils utilisent pour faire de l'histoire de la médecine. En effet, certains spécialistes n'hésitent pas à se servir des nouveaux outils psychiatriques et psychanalytiques pour analyser certains aspects de la folie dans les différents types de discours antiques.

C'est notamment le cas de Georges Devereux, pionnier de l'ethnopsychiatrie, qui propose une lecture des représentations poétiques des aberrations de l'âme à la lumière de la psychanalyse. Il publie ainsi, en 1976, une étude sur les rêves dans la tragédie grecque qu'il veut ethno-psycho-analyste et propose, dans un article, de considérer le dialogue final des Bacchantes d'Euripide comme une séance de psychothérapie psychanalytique où Cadmos tient le rôle de l'analyste et Agavé, celui de l'analysant, le père faisant peu à peu prendre conscience à sa fille, par le biais d'un entretien semidirectif, des actes horribles dont elle s'est rendue coupable alors qu'elle était possédée par Dionysos. L'ethnopsychiatrie instituée par Devereux considère que ce que l'époque contemporaine nomme psychiatrie possède son équi-

4 Pinel 1801.

Gesnerus 67 (2010)

99 
valent dans chaque culture humaine. Cette thèse se heurte, dès sa naissance dans les années 1970, aux critiques des tenants de la psychanalyse et de la psychiatrie qui affirment la supériorité de leurs savoirs scientifiques sur les savoirs et les coutumes anciennes voire primitives.

La plupart des études de ce genre ont été publiées dans les années 1970-1980, période où en Europe notamment, la psychiatrie évolue, se désinstitutionnalise donc se démocratise. Cette démocratisation et, de fait, la vulgarisation de certains aspects de la pensée psychiatrique ont vraisemblablement eu une incidence sur la manière d'interpréter l'histoire. Outre Devereux, ce parti pris dans la manière de traiter de la psychopathologie antique est également partagé par Bennett Simon qui, bien qu'en usant de plus de prudence, établit des analogies entre les différentes pathologies de la psyché évoquées ou décrites dans les discours médicaux, philosophiques et tragiques grecs, et la psychiatrie moderne. D'autres enquêtes proposant des parallèles entre la psychothérapie moderne et le fait psychique ancien ont été publiées sous forme d'articles comme ceux de Christopher Gill et d'Erling Eng ou encore comme celles de Paul Briot sur Cicéron qui tente de «psychanalyser» l'orateur à travers certaines de ses œuvres.

Il convient cependant de souligner le nombre restreint de discours de cette nature qui, bien que proposant une interprétation originale du fait psychopathologique antique, doivent néanmoins être lus avec la plus grande prudence tant la confusion des concepts, anciens et contemporains, peut être dangereuse et source de non-sens historiques. Si des parallèles peuvent en effet être établis entre certaines notions, certaines pathologies anciennes et actuelles, certaines situations psychiatriques, il semble cependant difficile d'insérer de la psychanalyse dans la poésie, la philosophie ou les discours médicaux antiques. Il s'agit donc de rester fidèle aux sources, de les interpréter avec toute la distance et la plus grande objectivité possible ce qu'a brillamment accompli Jackie Pigeaud dans son ouvrage sur La maladie de l'âme qui demeure, à ce jour, l'œuvre de référence sur la psychopathologie antique.

\section{La folie antique sous l'angle de la tradition médico-philosophique}

La publication de la thèse de Pigeaud en 1981 constitue un tournant majeur dans les recherches sur les représentations de la folie dans l'Antiquité grécoromaine. L'auteur analyse, à partir de l'étude des relations de l'âme et du corps dans les sources médicales et philosophiques, la formation, l'évolution et la fixation des concepts majeurs de la psychopathologie ancienne tels que 
la mélancolie, l'épilepsie, la manie, l'euthymie, depuis l'émergence de la médecine dite «scientifique», hippocratique, au $\mathrm{V}^{\mathrm{e}}$ siècle avant J.-C. jusqu'au II ${ }^{\mathrm{e}}$ siècle après J.-C. Il justifie l'emprunt de la formule «tradition médico-philosophique» à Pinel ${ }^{5}$ et le choix de son corpus de sources selon l'idée que les définitions de la maladie de l'âme chez les philosophes «apportent une connaissance positive et complètent le savoir médical antique» ${ }^{6}$. L'alliance du médical et du philosophique est ainsi présentée comme fondamentale voire indissociable, l'étude des maladies de l'âme ne pouvant être appréhendée sans comprendre les relations qui unissent la psyché au soma. Les écrits de Platon, Epicure, Asclépiade, Lucrèce, Cicéron, Sénèque constituent des documents de référence tandis que l'accent est mis, s'agissant de la tradition médicale, sur l'étude des réflexions de Galien, Caelius Aurelianus et Asclépiade. Le Corpus Hippocratique n'est pas oublié mais essentiellement utilisé comme référence permettant de saisir l'origine des concepts et leur première définition.

Cette vaste enquête qui rappelle que les discours médicaux, philosophiques et poétiques ne peuvent être envisagés comme relevant de domaines séparés mais complémentaires voire interdépendants est complétée et précisée par un ouvrage consacré au concept majeur de la manie, «sans doute le mot le plus général pour désigner la folie» ${ }^{7}$, à partir d'un corpus de sources circonscrit aux textes médicaux. Pigeaud démontre qu'il existe une «cassure dans le discours médical sur la folie» ${ }^{8}$ au cours de l'Antiquité gréco-romaine, rupture qui intervient notamment dans la seconde moitié du $\mathrm{II}^{\mathrm{e}}$ siècle avant J.-C. A ce moment, les concepts se stabilisent sous la «pression» des discours rhétorique et philosophique qui imposent une certaine contrainte de la définition. La mise en place d'une première classification des maladies, séparées entre chroniques et aiguës, permet de distinguer plus précisément les symptômes spécifiques de pathologies telles que la mélancolie, la manie, l'épilepsie, la phrénitis tout en considérant les liens qui existent entre ces affections. Bien que privilégiant les points de vue d'Arétée de Cappadoce et de Caelius Aurelianus cependant étudiés en regard de certaines observations hippocratiques, Pigeaud offre ainsi une histoire de l'évolution du concept de manie qui constitue une enquête de référence pour l'histoire de la médecine et la psychiatrie. En consacrant un dernier chapitre au traitement de la manie qui montre que la médecine se sert aussi d'apprentissages, d'expériences et de moyens qui débordent du champ médical strict, l'auteur ouvre la réflexion

5 Pinel 1801.

6 Pigeaud 1981, 15.

7 Pigeaud 1987, 29.

8 Pigeaud 1987, 221. 
et des perspectives sur une représentation sociale et culturelle de la folie. Celle-ci est cependant difficile à établir car le malade et sa perception de la maladie, ses ressentis sont rarement évoqués dans les sources médicales. Quand bien même les recherches de Pigeaud n'embrassent pas tous les discours antiques mais se limitent au corpus, déjà très vaste, des sources médicales et philosophiques, La maladie de l'âme et Folie et cures de la folie constituent les seules enquêtes aussi exhaustives menées à ce jour sur les aberrations de l'âme dans l'Antiquité gréco-romaine.

Bien entendu, des précédents existent consistant en travaux ciblés sur des pathologies spécifiques telles que la mélancolie ou l'épilepsie. L'enquête de Helmut Flashar, qui reprend et précise celle de Raymond Klibansky, Erwin Panofsky et Fritz Saxl, est vraisemblablement l'analyse la plus complète menée sur la mélancolie. Cette affection de l'âme n'a ni limites temporelles ni frontières disciplinaires et fascine quelles que soient les époques de sorte qu'on ne peut guère s'étonner que, de toutes les pathologies de la raison, elle est celle qui a donné lieu au plus grand nombre de recherches pour la période antique. Des discours philosophiques et médicaux, le Problème $X X X$ attribué au Pseudo-Aristote, qui traite des liens entre le tempérament mélancolique et le génie, est celui qui a fait l'objet des plus nombreuses interprétations de la part des modernes comme en témoignent les études de Pierre Gravel, Fabrice Roussel et, plus récemment, de Pigeaud, Philip Van Der Eijk et Jacques Jouanna. Walter Müri, en analysant précisément la question humorale, a précisé les liens existants entre l'affection mélancolique et l'humeur bile noire et, de fait, les rapports entre la physiologie et la psychopathologie. L'épilepsie est, elle aussi, souvent analysée dans sa dimension médicale, hippocratique notamment, avec le traité de la Maladie sacrée qui, comme le rappelle Owsei Temkin, est la première étude sur l'épilepsie que l'on possède. Le dessein premier de la réflexion hippocratique n'est pourtant pas de révéler le fonctionnement du processus cognitif ou des mécanismes de la maladie mais de défendre le principe selon lequel toutes les affections ont des causes physiologiques, «naturelles» contre ceux qui abusent de la foi du peuple en lui faisant croire qu'elle est la marque d'un châtiment divin. Des articles et études récentes ont revisité ce traité, telles que celles de Van Der Eijk, d'Amneris Roselli et de Julie Laskaris. Outre les Hippocratiques, Aristote livre également une réflexion singulière sur les mécanismes du «grand mal» dans le petit traité Sur le sommeil que l'analyse très pertinente d'Armelle Debru permet d'expliciter. La pathologie épileptique antique a donc essentiellement étudiée à partir de traités spécifiques, médicaux et philosophiques. Il convient cependant de citer les travaux de Horst Schneble et, plus récents, de Michael Wohlers, qui proposent une représentation de 
cette affection dans une perspective plus large, combinant les approches médicales et socioculturelles.

Les sources médico-philosophiques constituent des documents fondamentaux pour appréhender la psychopathologie antique. On peut s'étonner voire regretter que la terminologie des troubles de la raison, des états inhabituels de l'âme n'aient pas donné lieu à des études plus approfondies tout en saluant les approches intéressantes de Simon Byl sur le délire, de Danielle Gourevitch et d'Yves Pelicier sur le vocabulaire latin de la folie. Ce type d'enquête est d'autant plus intéressant qu'il renvoie de fait à la question des frontières antiques de la notion de folie, problème complexe et ambigu dont on a encore bien du mal aujourd'hui, en dépit d'une classification de plus en plus précise des pathologies, à en fixer les limites précises. Si la psychopathologie antique bénéficie donc d'une bibliographie médico-philosophique relativement abondante, il est cependant dommageable que cette dimension spécifique soit encore si dissociée de l'aspect social et culturel de la folie. Les enquêtes publiées ces dernières années démontrent cependant qu'il constitue un axe de recherche en pleine expansion.

\section{Vers une histoire sociale de la psychopathologie antique?}

Il va de soi que la folie n'est pas seulement appropriée et perçue par les médecins et les philosophes. Elle est avant tout vécue, au quotidien, d'abord par ceux qui en souffrent mais aussi par l'ensemble de la communauté qui en est témoin et qui participe d'une certaine manière à l'intégration et/ou à l'exclusion du fou en son sein. Il existe donc nécessairement, pour reprendre la formule foucaldienne, un «geste social» à l'égard du fou et, de fait, une empreinte culturelle de la folie au sein de la communauté.

Publiée en 1894, la vaste enquête d'Erwin Rohde sur les représentations de la psyché et le culte de l'âme dans le monde grec offre une première analyse de la dimension socioculturelle de la psychopathologie ancienne. En abordant la religion dionysiaque, il fait le point sur le concept de la «mania divine», et ouvre sur une représentation de la folie que l'on serait tenté de nommer «irrationnelle» mais qui n'est pas pour autant perçue de cette manière par les Anciens. Si les médecins avancent une explication physiologique du délire, il est traditionnellement admis que la folie a des causes «divines» en cela qu'elle relève de l'intervention d'une puissance supérieure qui, en prenant possession de la raison d'un individu, le plonge dans un état d'enthousiasme dont l'orgiasme dionysiaque constitue un des meilleurs exemples. Cette possession souhaitée a une fonction cathartique, elle est 
considérée comme le traitement par excellence de la manie. Cette conception de la folie et de son traitement préexiste à la représentation médicale «rationnelle» et ne disparaît pas avec la diffusion de la pratique «scientifique» mais existe en parallèle voire en complément de cette dernière. On touche là un des aspects essentiels des mentalités antiques et l'erreur serait de dissocier ou d'opposer de telles pratiques selon une dichotomie rationnel/ irrationnel car il ne fait nul doute que les Anciens ont eu recours à ces différents moyens de traitement du délire pour tenter de le guérir.

Après Rohde, il faut attendre l'ouvrage d'Eric R. Dodds, publié en 1949, qui, dans un chapitre sur Les bienfaits de la folie, revient sur les aspects spécifiques de la possession en rappelant combien la représentation divine du délire est celle partagée par le plus grand nombre. Il y évoque également la division quadripartite de la manie développée par Platon dans Phèdre. De nombreuses études ont été menées sur cette conception platonicienne de la manie, celles d'Ivan Linforth et de Luc Brisson se présentant comme des enquêtes de référence. Pour la manie télestique, on ne peut passer outre l'ouvrage de Henri Jeanmaire qui est vraisemblablement à ce jour l'étude la plus complète publiée sur le culte dionysiaque, des origines à sa diffusion dans le monde gréco-romain. L'auteur y analyse également, à fin de comparaison avec les rituels orgiaques anciens, les manifestations contemporaines de la possession. Ce parallèle instructif est également proposé, dans la lignée des travaux de Michel Leiris sur lesquels s'appuie Jeanmaire, par Gilbert Rouget qui précise les relations spécifiques que l'orgiasme antique, grec notamment, entretient avec la musique, le son et le rythme et son importance dans la mise en transe des fidèles participant à ces cultes.

Cette représentation «traditionnelle» de la folie comme possession divine est également mise en scène dans le théâtre tragique et comique d'Eschyle à Sénèque. Cette littérature a été le point de départ de nombreuses études cependant plus souvent axées sur des questions de stylistique et de linguistique que sur ce que ce type de sources est susceptible de révéler de la réalité socioculturelle du délire. Dans la poésie, la manie est présentée comme une possession douloureuse, non souhaitée, expression d'un châtiment divin suite à une faute ou un manquement commis à l'égard d'un dieu ou d'une déesse. Au-delà des métaphores et des symboles, cette représentation a un ancrage dans la réalité antique: les troubles mis en scène, les altérations du raisonnement et le comportement propre à la possession sont identiques à ceux qui se manifestent lors des rituels voire à ceux consignés dans les écrits médicaux. Si ces textes poétiques doivent être interprétés avec la plus grande prudence du fait de leur nature fictive, ils permettent néanmoins d'établir des parallèles avec d'autres sources contribuant ainsi à préciser la conception 
antique du délire. Cependant, outre les ouvrages de Ruth Padel et les rapports effectués entre les discours poétiques et médico-philosophiques par Pigeaud et Thomas Rütten, aucune véritable étude traitant de la psychopathologie des Anciens dans sa dimension sociale n'a été menée. Celle-ci est difficile à saisir et apparaît le plus souvent en contre-jour de certains discours. Ainsi, en évoquant les différents états du mélancolique, ses rapports avec le génie politique ou artistique, le Pseudo-Aristote livre quelques clés s'agissant de l'intégration de ce type d'individu au sein de la cité, clés qui peuvent être complétées par les Lettres d'Hippocrate, échangées entre le médecin et les représentants de la cité d'Abdère au sujet de la prétendue folie de Démocrite. Yves Hersant identifie dans ces échanges épistolaires la convergence de trois traditions complémentaires: la réflexion de Démocrite sur la composition de l'univers et la place de l'homme au sein du cosmos, la théorie des Hippocratiques sur les mouvements et les effets de la bile noire et, enfin, la tradition aristotélicienne du Problème $X X X$ qui assimile la mélancolie à une forme de génie. Ces sources sont précieuses car elles permettent de déceler ce que Foucault nomme «le geste social» à l'égard du fou, la façon dont la société le considère et se comporte par rapport à lui. L'affection de Démocrite que les Abdéritains nomment manie dans les Lettres, Hippocrate la désigne comme mélancolie et l'on peut ainsi se demander de quelle réalité socioculturelle est porteuse une telle distinction dénominative. La tristesse, le mal de vivre et les angoisses mis en scène dans le théâtre et la poésie peuvent ainsi être perçues comme autant de manifestations de la mélancolie, comme le pensent notamment Jean Starobinski et, plus récemment, Pigeaud qui rappellent combien ce trouble de l'âme constitue souvent le fil du génie poétique antique.

S'il apparaît nécessaire de comparer et de panacher les différents discours sur la folie pour mieux appréhender le fait psychopathologique dans une large dimension socioculturelle, il convient également de les confronter, lorsque cela est possible, aux aspects plus concrets parfois offerts par l'archéologie. La découverte d'amulettes apotropaïques destinées à protéger celui qui les porte de l'épilepsie, de datation souvent tardive (III ${ }^{\mathrm{e}}$ siècle après J.-C.), permet de constater la permanence des conceptions qui associent la maladie à une malédiction divine. De la même manière, quelques inscriptions propitiatoires des temples permettent de dégager la conception «ordinaire» de la folie et les différents moyens auxquels ont recours les individus, tout au long de la période gréco-romaine, pour la prévenir, la traiter et la guérir. Cette documentation est rare et lacunaire et, là encore, les comptes rendus des découvertes demeurent souvent centrés sur l'étude de l'objet archéologique lui-même et moins sur ce qu'il témoigne de la réalité socioculturelle. 
Il convient cependant de saluer les initiatives de Van Der Eijk et l'enquête d'Angelo Chaniotis qui contribuent à développer cet axe de recherche en appréhendant la médecine ancienne dans un plus large contexte social et culturel. J'adjoins également à ces enquêtes récentes mon travail de thèse sur les représentations du délire à l'époque classique qui montre à partir de l'analyse d'un éventail de sources de nature très variée et de l'étude de la sémantique que les frontières sont ténues entre les différents types de discours et tente, en partant de ce constat, de restituer la représentation commune de la folie, dans sa réalité culturelle et sociale.

\section{Conclusion}

Si les discours antiques sur la folie sont relativement nombreux et de nature très variée, ils souffrent cependant de l'interprétation souvent segmentée, strictement délimitée à un champ disciplinaire, qu'en livrent les modernes. Bien qu'il convient de distinguer, dans la psychopathologie antique, les territoires respectifs de la philosophie, de la médecine et de la littérature poétique et de les étudier pour l'éclairage spécifique qu'ils apportent, on peut regretter qu'ils ne soient pas confrontés et comparés de manière plus systématique, ne serait-ce que de manière partielle par l'analyse précise d'un trouble de l'âme ou d'un terme relatif au délire, afin de réintégrer la question de la folie dans un plus large contexte socioculturel.

Les ouvrages de Pigeaud demeurent incontestablement les enquêtes les plus exhaustives menées sur la question des maladies de l'âme dans l'Antiquité gréco-romaine, et si l'auteur a su établir des parallèles entre différents types de discours, son analyse, aussi vaste et précieuse soit-elle pour l'histoire de la psychiatrie, demeure profondément axée sur les sources médico-philosophiques qui ne permettent pas, à elles seules, d'élucider les questions relatives à la place du fou dans la société notamment. Certains discours sont encore à exploiter comme les textes juridiques, susceptibles de fournir, par ce qu'ils disent ou taisent, des informations capitales propre à nourrir un axe de recherches «sociales» en développement qui viendrait compléter les enquêtes déjà menées. Il convient de ne pas oublier que la psychopathologie antique est un champ historiographique relativement récent qui s'étoffe progressivement d'études riches contribuant indéniablement à la construction d'une histoire de la folie antique dans la lignée des écrits foucaldiens. 


\section{Bibliographie sélective}

\section{Sources}

Alexandre de Tralles, Euvres médicales, introduit et traduit par Félix Brunet, 4 volumes (Paris 1933-1937)

Anonyme latin, Traité de physiognomonie, traduit par Jacques André (Paris 1981)

Arétée, Traité des signes, des causes et de la cure des maladies aiguës et chroniques, traduit par M. L. Renaud (Paris 1834)

Aristophane, Les Guêpes, La paix, texte établi par Victor Coulon et traduit par Hilaire Van Daele (Paris 1924)

Aristote, «De la divination dans le sommeil», traduit par Jackie Pigeaud, dans: Aristote, La vérité des songes (Paris 1995)

- «De l'âme», texte établi par Antonio Jannone et traduit par Edmond Barbotin (Paris 1966)

- «Ethique à Nicomaque», traduit par Jean Tricot (Paris 1959)

- «Petits traités d'histoire naturelle», traduit par René Mugnier (Paris 1953)

- «Problème XXX,1», traduit par Jackie Pigeaud, dans: Aristote, l’homme de génie et la mélancolie (Paris 1991)

- «Problèmes», Tomes I à III, traduit par Pierre Louis (Paris 1991-1994)

Asclépiade, Asclepiadis Bithyni Fragmenta, édité par Christianus Gottlieb Gumpert (Weimar 1794)

Caelius Aurelianus, De acutis et chronicis morbis, On Acute Diseases and on Chronic Diseases, édité et traduit par Israel E. Drabkin (Chicago 1950)

Celse, De medicina, traduit par W. G. Spencer, 3 volumes (Oxford 1971-1979)

Cicéron, De la divination, traduit et commenté par Gérard Freyburger et John Scheid (Paris 1992)

- The Nature of Gods, traduit par Patrick Gerard Walsh (Oxford 1998)

- Tusculanes, texte établi par Georges Folhen et traduit par Jules Humbert, 2 volumes (Paris 1931)

Démocrite, Doctrines philosophiques et réflexions morales, traduit par Maurice Solovine (Paris 1928)

Eschyle, Les Suppliantes, Les Perses, Les Sept contre Thèbes, Prométhée enchaîné, traduit par Paul Mazon (Paris 1920)

- Agamemnon, Les Choéphores, Les Euménides, traduit par Paul Mazon (Paris 1925)

Euripide, Hippolyte, Andromaque, Hécube, traduit par Louis Méridier (Paris 1927)

- Héraclès, Les suppliantes, Ion, traduit par Louis Parmentier et Henri Grégoire (Paris 1923)

- Les Bacchantes, traduit par Henri Grégoire (Paris 1961)

- Les Bacchantes, traduit par Jeanne Roux, Vol. I-II (Paris 1970-1972)

- Oreste, traduit par Louis Méridier (Paris 1959)

Galien, In Hippocratis epidemiarum, livres I-VI, édité et traduit par Ernst Wenkebach et Franz Pfaff (Leipzig/Berlin 1934-1956)

- L'âme et ses passions; les passions et les erreurs de l'âme; les facultés de l'âme suivent les tempéraments du corps, introduit, traduit et annoté par Vincent Barras, Terpsichore Birchler et Anne-France Morand (Paris 1995)

- On the Natural Faculties, traduit par A. J. Brock (Oxford 1916)

- Scripta minora, volumes I et II, édité par Johannes Marquart et Iwan von Müller (Leipzig 1884-1891)

Hippocrate, Euvres complètes, volumes 1 à 10, traduit et commenté par Emile Littré (Paris 1839-1861)

Platon, Ion, Ménexène, Euthydème, traduit par Louis Méridier (Paris 1931)

- Hippias mineur, Alcibiade, Apologie de Socrate, Euthyphron, Criton, traduit par Maurice Croiset (Paris 1920)

- Phèdre, traduit par Léon Robin (Paris 1933)

- Le banquet, traduit par Léon Robin (Paris 1929)

- La République, I-X, traduit par Emile Chambry (Paris 1932/1933)

- Les lois, III-VI, traduit par Edouard des Places (Paris 1951)

- Les lois, VII-X, traduit par Auguste Diès (Paris 1956) 
- Le politique, traduit par Auguste Diès (Paris 1935)

- Timée, Critias, traduit par Albert Rivaud (Paris 1925)

Rufus d'Ephèse, Euvres, édité et traduit par Charles Daremberg et Charles Emile Ruelle (Paris 1879)

Sénèque, Dialogues, 4 volumes, édité et traduit par René Waltz et Abel Bourgery (Paris 19221927)

- Lettres à Lucilius, 5 volumes, édité et traduit par François Préchac et Henri Noblot (Paris 1945-1964)

- Questions naturelles, 2 volumes, édité et traduit par Paul Oltramare (Paris 1961)

- De la clémence, traduit par François-Régis Chaumartin (Paris 2005)

- Tragédies, 3 volumes, édité et traduit par François-Régis Chaumartin (Paris 1996-1999)

- Phaedra, texte et commentaire par Pierre Grimal (Paris 1965)

Sophocle, Ajax, traduit par Paul Mazon (Paris 1958)

- Antigone, traduit par Paul Mazon (Paris 1955)

\section{Auteurs modernes}

Albrecht, M. von, «Il teatro di Seneca e la semiotica della follia», Dionisio 54 (1983) 117-125

Ando, Valeria, «Vin et mania», dans: Fournier, Dominique/Salvatore D'Onogrio (éds), Le ferment divin (Paris 1991) 167-179

Annecchino Manni, M., «Follia ed eroismo nell'Aiace Sofocleo», Rivista di Studi Classici 26 (1978) 459-465

Assaël, Jacqueline, «Sunesis dans Oreste d'Euripide», L'Antiquité Classique 65 (1996) 53-69

Ballériaux, Omer, «Mantique et télestique dans le Phèdre de Platon», Kernos 3 (1990) 35-43

Bataille, Marie-Josée, «Le fou et le devin dans la tragédie grecque», Cahiers du GITA 4 (1988) 147-159

Baumann, E. D., «Die pseudohippokratische Schrift 〈peri manies»», Janus 42 (1938)

Belis, Annie, «Musique et transe dans le cortège dionysiaque», Cahiers du GITA 4 (1988) 9-29

Beta, Simone, «Madness on the comic stage», Greek Roman and Byzantine Studies 40 (1999) 135-158

Billault, Alain, «La folie poétique», Bulletin de l'Association Guillaume Budé 61 (2002) 18-35

Bourguignon, Erika, Religion, Altered States of Consciousness and Social Change (Columbus, Ohio, 1973)

Bremmer, Jan, The Early Greek Concept of Soul (Princeton 1983)

Briot, Paul, «Deux remarques sur la psychologie de Cicéron», Latomus 25 (1966) 743-755

- «Cicéron, approches d'une psychanalyse», Latomus 28 (1969) 1040-1049

- «Traces névrotiques chez Cicéron», Latomus 32 (1973) 595-605

Brisson, Luc, «Platon psychanalysé», Revue des Etudes Grecques 86 (1973) 224-231

- «Du bon usage du dérèglement», dans: Jean-Pierre Vernant et al. (éds), Divination et rationalité (Paris 1974) 220-248

Bruun, Henriette, «〈De morbo sacro〉 et 〈De aere aquis locis»», Classica et mediaevalia 48 (1997) 115-148

Byl, Simon, «Le délire, symptôme cnidien ou coaque?», dans: Jouanna, Jacques/Jean Leclant (éds), La médecine grecque antique, Actes du 14e colloque de la villa Kérylos (Paris 2004) $45-52$

- «Le délire hippocratique dans son contexte», Revue Belge de Philologie et d'Histoire 84 (2006) $5-24$

Calvo Martinez, José Luis, «Sobre la «mania〉 y el entusiasmo», Emerita 41 (1973) 157-182

Cavada, Enrico/Gianfranco Paci, «Un amuleto contro l'epilessia dall'alto garda», Archeologia Classica 53 (2002) 221-255

Centari, M., «Nome del male: 〈Phrenitis〉 e <epilepsia〉 nel Corpus Galenicum», Museum Patavium 5 (1988-1989) 47-79

Chaniotis, Angelo, «Illness and cures in the Greek propitiatory inscriptions and dedications of Lydia and Phrygia», dans: Van Der Eijk, Philip/Herman Frederik Johan Horstmanshoff/ P. H. Schrijvers (eds), Ancient Medicine in its Socio-cultural Context (Amsterdam 1995) 323-344

108 Gesnerus $67(2010)$ 
Charlier, P., «Les folies d'Héraclès. Rage fureur et délire chez le héros thébain d'Euripide à Sophocle», Medicina nei secoli 15 (2003) 595-613

Chirassi Colombo, Ileana, «Le Dionysos oraculaire», Kernos 4 (1991) 205-218

Ciani, Maria Grazia, «Lessico e funzione della follia nella tragedia greca», BIFG (1974) 70-110

Corleto, Luigia Melillo, «The Concept of Mania in Greek Medical and Philosophical Literature», Medicina nei Secoli 4 (1992) 33-42

Croissant, Jeanne, Aristote et les mystères (Genève 1932)

Czerwinska, Jadwiga, «Hercules Furens. An Euripidean allegory of human madness», Eos 92 (2005) 161-182

Darbo-Peschanski, Catherine, «La folie pour un regard. Oreste et les divinités de l'échange (Erinyes, Euménides, Charites)», Métis 4 (2006) 13-28

Debru, Armelle, «L'épilepsie dans le 〈De Somno〉 d'Aristote», dans: Sabbah, Guy (éd.), Médecins et médecine dans l'Antiquité (Saint-Etienne 1982) 25-41

Devereux, Georges, Dreams in Greek Tragedy (Oxford 1976)

- «The psychotherapy scene in Euripide's 〈Bacchae»», Journal of Hellenic Studies 60 (1970) 35-48

Dodds, Eric Robertson, Les Grecs et l'irrationnel (Paris 1965)

Dorion, Louis-André, «Socrate, le «daimonion〉 et la divination», dans: Laurent, Jérôme (éd.), Les dieux de Platon (Caen 2003) 169-192

Drabkin, Israel E., «Remarks on Ancient Psychopathology», Isis 46 (1955) 223-234

Dumortier, Jean, Le vocabulaire médical d'Eschyle et les écrits hippocratiques (Paris 1935)

Edelstein, Ludwig/Emma Edelstein, Asclepius, Collection and Interpretation of the Testimonies (Baltimore 1945)

Eng, Erling, «Modern psychotherapy and ancient rhetoric», Psychotherapy and Psychosomatics (1974) 493-496

Feier, Isaia, L'Eros platonicien (Jerusalem 1990)

Filhol, Emmanuel, «Heracleie nosos, l'épilepsie d'Héraclès», Revue d'histoire des religions 206 (1989) 3-20

Flacelière, Robert, «Le délire de la pythie est-il une légende?», Revue des études anciennes 51 (1950) 306-324

Flashar, Helmut, Melancholie und Melancholiker in den medizinischen Theorien der Antike (Berlin 1966)

Foucault, Michel, Histoire de la folie à l'âge classique (Paris 1961)

Gallini, Clara, «La folia panica», dans: Studi e materiali di storia delle religioni 32 (1961) 205-236

Garcia Gual, Carlos, «Del melancolico como atrabilario. Segun las antiguas ideas griegas sobre la enfermedad de la melancolia», Faventia 6 (1984) 41-50

Garzya, Antonio, «Sunesis como malattia, Euripide e Ippocrate», dans: Lopez Ferez, Juan Antonio (éd.), Tratados Hippocraticos, Actas del VII e colloque international hippocratique (Madrid 1992) 505-512

Gill, Christopher, «Ancient Psychotherapy», Journal of the History of Ideas 46 (1985) 307-325

Gold, Barbara K., «Dionysos, Greek Festivals and Treatment of Hysteria», Laetaberis 6 (1988) $16-28$

Gotshalk, Richard, Loving and Dying: a Reading of Plato's Phaedo, Symposium and Phaedrus (Lanham 2001)

Gourevitch, Danielle, «Médecins fous», L'Evolution psychiatrique 47 (1982) 1113-1118

- «Les mots pour dire la folie en latin: à propos de passages de Celse et de Caelius Aurelien», L'Evolution psychiatrique 56 (1991) 561-568

Gravel, Pierre, «Aristote, sur le vin, le sexe, la folie, le génie. Mélancolie», Etudes françaises 18 (1982) 129-185

Gunder, Beate, «Soma and Psyche in Hippocratic Medicine», dans: Wright, John P./Paul Potter (eds), Psyche and Soma: Physicians and Metaphysicians on the Mind-Body Problem from Antiquity to Enlightenment (Oxford 2000) 13-35

Hanson, Anne Ellis, «Uterine Amulets and Greek Uterine Medicine», Medicina nei Secoli 7 (1995) 281-299

Helm, Robert Meredith, «Psyche and Soma, Mind and Body in Greek Philosophy and Modern Medicine», dans: Boudouris, Konstantinos (ed.), Philosophy and Medicine, Ninth International Conference on Greek Philosophy (Alimos 1998) 88-94 
Hersant, Yves, Hippocrate, sur le rire et la folie (Paris 1991)

Horstmanshoff, Herman Frederik Johan/Stol Marten, Magic and Rationality in Ancient Near Eastern and Graeco-Roman Medicine (Leyden 2004)

Huber, Jean Paul, «Arétée de Cappadoce et la psychose maniaco-dépressive», L'information psychiatrique 61 (1985) 1373-1386

Jacques, Jean-Marie, «La bile noire dans l'Antiquité grecque: médecine et littérature», Revue des Etudes Anciennes 100 (1998) 217-234

Jeanmaire, Henri, Dionysos, histoire du culte de Bacchus (Paris 1951)

- «Le traitement de la mania dans les 〈Mystères〉 de Dionysos et des Corybantes», Journal de Psychologie normale et pathologique 42 (1949) 64-82

Joly, Robert, «La question hippocratique et le témoignage du Phèdre», Revue des Etudes Grecques 74 (1961) 69-92

Jouanna, Jacques, «La théorie de l'intelligence et de l'âme dans le traité hippocratique du Régime: ses rapports avec Empédocle et le Timée de Platon», Revue des Etudes Grecques 79 (1966) 15-18

- «La maladie sauvage dans la collection hippocratique et la tragédie grecque», Métis 3 (1988) 343-360

- «Aux racines de la mélancolie: la médecine grecque est-elle mélancolique?», dans: Clair, Jean/Robert Kopp (éds), De la mélancolie (Paris 2007) 11-51

Klibansky, Raymond/Erwin Panofsky/Fritz Saxl, Saturne et la mélancolie, études historiques et philosophiques: nature, religion, médecine et art (Paris 1989)

Kotansky, Roy, «Two Amulets in the Getty Museum», Getty Museum Journal 8 (1980) 180-188

Lanata, Giuliana, Medicina magica e religione popolare in Graecia fino all'eta di Ippocrate (Rome 1967)

Laskaris, Julie, The Art is Long. On the Sacred Disease and the Scientific Tradition (Leyden 2002)

Le Person, Gwenaëlle, La psukhê et les phrenes sont malades. Représentations du délire à l'époque classique $\left(V I^{e}-I I I^{e}\right)$, Thèse de l'Université de Rennes 2 (2007)

- «Le portrait du mélancolique dans les Problèmes du Pseudo-Aristote et les traités aristotéliciens», dans: Dasen, Véronique et al. (éds), Langages et métaphores du corps dans le monde antique (Rennes 2008)

Linforth, Ivan M., «Telestic Madness in Plato, Phaedrus 244 d-e», University of California Publications in Classical Philology 13 (1946a) 163-172

- «The corybantic rites in Plato», dans: University of California Publications in Classical Philology 13 (1946b) 140-144

Lippi, Donatella, «About a medical amulet in the Archeological Museum of Florence», Medicina nei Secoli 2 (1990) 215-220

Luck, G., «Was Lucretius really mad?», Euphrosyne 16 (1988) 289-294

Magnien, Victor, «Quelques mots du vocabulaire grec exprimant des opérations ou des états de l'âme», Revue des Etudes Grecques 40 (1927) 117-141

Martinez Conesa, Jose A./M. A. Corbera Lloveras, «La perturbacion mental expresada con 〈phrenes〉 y «mania〉 en las tragedias de Euripides», Helmantica 44 (1993) 37-49

Mazzini, Innocenzo, «Il folle da amore», dans: Alonso Stefania et al., (éds), Il poeta eligiaco e il viaggio d'amore. Dall'innamoramento alla crisi (Bari 1990) 39-83

- «Didone abbandonata: innamorata o pazza? La psichiatria antica, una chiave di lettura per il IV libro dell'Eneide», Latomus 54 (1995) 92-105

Michel, Jacques-Henri, «La folie avant Foucault, furor et ferocia», L'Antiquité classique 50 (1981) $517-525$

Minois, Georges, Histoire du mal de vivre: de la mélancolie à la dépression (Paris 2003)

Moore, J. D., «The Philosopher's frenzy», Mnemosyne 22 (1969) 225-230

Moreau, Alain, «Transes douloureuses dans le théâtre d'Eschyle», Cahiers du GITA 4 (1988) 103-114

Müri, Walter, «Melancholie und schwarze Galle», Museum Helveticum 10 (1953) 21-38

Musitelli, S., «Riflessi di teorie mediche nelle Baccanti di Euripide», Dionisio 42 (1968) 93-114

Nicholson, Graeme, Plato's Phaedrus: The Philosophy of Love (Western Lafayette, IN, 1999)

Orlandini, Anna, «Parmi les noms latins de l'épilepsie 〈Morbus maior»», dans: Debru, Armelle/ Guy Sabbah (éds), Nommer la maladie. Recherches sur le lexique gréco-latin de la pathologie (Saint-Etienne 1998) 83-91 
Padel, Ruth, In and Out of the Mind. Greek Images of the Tragic Self (Princeton 1992)

- Whom Gods Destroy (Princeton 1995)

Pelicier, Yves, «Stability in the meaning of the Latin vocabulary for 〈folie〉 (insanity)», dans: Zeitschrift für Klinische Psychologie und Psychotherapie 30 (1982) 68-76

- «Les sources classiques du vocabulaire de la folie», dans: Breton, Stanislas et al. (éds), $D u$ banal au merveilleux, Mélanges offerts à Lucien Jerphagnon (Fontenay/Saint-Cloud 1989) 253-273

Pigeaud, Jackie, La maladie de l'âme: étude sur la relation de l'âme et du corps dans la tradition médico-philosophique antique (Paris 1981)

- Folies et cures de la folie chez les médecins de l'antiquité gréco-romaine, la manie (Paris 1987)

- Aristote, l'homme de génie et la mélancolie (Paris 1991)

- De la mélancolie, fragments de poésie et d'histoire (Paris 2005)

- «Euripide et la connaissance de soi. Quelques réflexions sur Hippolyte», Les Etudes Classiques 44 (1976) 3-24

- «Une physiologie de l'inspiration poétique: de l'humeur au trope», Les Etudes Classiques 46 (1978) 23-34

- «Quelques aspects du rapport de l'âme et du corps dans le Corpus Hippocratique et la tradition médico-philosophique», dans: Grmek, Mirko D. et al. (éds), Hippocratica, Actes du colloque hippocratique de Paris (Paris 1980) 417-432

- «L'Antiquité classique et les débuts de la psychiatrie française», dans: Postel, Jacques/Claude Quétel (éds), Nouvelle histoire de la psychiatrie (Toulouse 1983) 129-145

- «Prolégomènes à une histoire de la mélancolie», Histoire, économie et société 3 (1984a) 501-510

- «De la mélancolie et de quelques autres maladies dans les Etymologies IV d'Isidore de Séville», Mémoires du Centre Jean Palerne 5 (Saint-Etienne 1984b) 87-109

- «La folie dans la satire II, 3 d'Horace», Orphea Voce 3 (1990) 7-43

- «Mesure et mélancolie», dans: Clair, Jean/Robert Kopp (éds), De la mélancolie (Paris 2007) 53-73

Pinel, Philippe, Traité médico-philosophique sur l'aliénation mentale (1801, repr. 1965)

Pralon, Didier, «Qu'est-ce que la folie? Qu'est-ce que la santé mentale? Hippocrate et Démocrite (Lettres 18 et 20)», dans: Boulhol, Pascal/Françoise Gaide/Mireille Loubet (éds), Guérisons du corps et de l'âme. Approches pluridisciplinaires (Aix-en-Provence 2006) $15-27$

Prigent, Hélène, «Mélancolie antique, une philosophie de l'image?», dans: Clair, Jean/Robert Kopp (éds), De la mélancolie (Paris 2007) 75-93

Printz, Othon, «Quelques réflexions sur l'épilepsie à partir des récits de la guérison de l'enfant épileptique», Revue d'histoire et de philosophie religieuse 82 (2002) 391-400

Puccini, Géraldine, «La folie amoureuse dans les Métamorphoses d'Apulée», Bulletin de l'Association Guillaume Budé 4 (1998) 318-336

Quétel, Claude, «La folie à la frontière de l'âme et du corps», Clio Medica 19 (1984) 159-163

Renier, E., «Observations sur la terminologie de l'aliénation mentale», Revue internationale des droits de l'Antiquité 5 (1950) 429-455

Rohde, Erwin, Psyche. Le culte de l'âme chez les Grecs et leur croyance en l'immortalité (Paris 1928)

Roselli, Amneris, La malattia sacra (Venise 1996)

Rosetti, Livio, Understanding the Phaedrus. Proceedings of the II Symposium platonicum (Sankt Augustin 1992)

Rouget, Gilbert, La musique et la transe, esquisse d'une théorie générale des représentations de la musique et de la possession (Paris 1980)

Roussel, Fabrice, «Le concept de la mélancolie chez Aristote», RHS 41 (1988) 299-330

Rütten, Thomas, Demokrit-lachender Philosoph und sanguinischer Melancholiker. Eine pseudohippokratische Geschichte (Leyden 1992)

Salem, Jean, La légende de Démocrite (Paris 1996)

Sarantoglou, Yorgos E., «Fonction dramaturgique du fait pathopsychologique dans l'Ajax de Sophocle», Littérature, Médecine et Société 5 (1983) 55-67

- Quelques réflexions psychopathologiques et psychothérapeutiques sur la 〈folie〉 dans la tragédie grecque: le 〈cas〉 de l’Ajax sophocléen. Mémoire de psychiatrie (Nantes 1990) 
Schneble, Horst, Krankheit der ungezählten Namen. Ein Beitrag zur Sozial-, Kultur- und Medizingeschichte der Epilepsie anhand ihrer Benennungen vom Altertum bis zur Gegenwart (Bern 1987)

Sidwell, Keith, «Was Philocleon cured?», Classica et Mediaevalia 41 (1990) 3-31

Simon, Bennett, Mind and Madness in Ancient Greece. The Classical Roots of Modern Psychiatry (Londres 1984)

Simpson, Michael, «Sophocles’ Ajax: his madness and transformation», Arethusa 2 (1969) 88-103

Starobinski, Jean, Histoire du traitement de la mélancolie des origines jusqu'à 1900 (Bâle 1960)

- «L'épée d'Ajax», dans: ibid., Trois Fureurs (Paris 1974)

- «Démocrite parle», Le débat 29 (1984) 49-72

Stegen, Guillaume, «La folie de Phèdre», L'Antiquité Classique 34 (1970) $443-449$

Stock, F., «Concetto e trattamento dell'insania in A. Cornelio Celso», Studi di Filologi e lettera a cura della faculta di lingue e lettera dell'Universita degli studi di Pisa 4 (1980) 9-42

Sullivan, Shirley Darcus, Psychological and Ethical Ideas. What Early Greeks Say (Leyden 1995a)

- Aeschylus's use of psychological terminology, traditional and new (Montreal 1995b)

- Sophocle's use of psychological terminology, old and new (Montreal 1999)

- Euripide's use of psychological terminology (Montreal 2000)

Taldone, Antonietta, «Su insania e furor in Cicerone», Bollettino di Studi Latini 23 (1993) 3-19

Temkin, Owsei, The Falling Sickness: A History of Epilepsy from the Greeks to the Beginning of Modern Neurology (Baltimore 1945)

- «The doctrine of epilepsy in the Hippocratic writings», Bulletin d'Histoire Médicale 1 (1933) 277-322

- «Hippocrates as the Physician of Democritus», Gesnerus 57 (1985) 455-464

Van Der Eijk, Philip (ed.), Ancient Histories of Medicine. Essays on Medical Doxography and Historiography, dans: Classical Antiquity (Leyden 1999)

- «The 〈theology> of the Hippocratic treatise On the Sacred Disease», Apeiron 23 (1990) 87-119

- «Aristotle's Psycho-physological Account of the Soul-Body Relationship», dans: Wright, John P./Paul Potter (eds), Psyche and Soma: Physicians and Metaphysicians on the MindBody Problem from Antiquity to Enlightenment (Oxford 2000) 57-77

- «Aristotle on Melancholy», dans: Medicine and Philosophy in Classical Antiquity: Doctors and Philosophers on Nature, Soul, Health and Disease (Cambridge 2005) 139-168

Vicaire, Paul, «Platon et la divination», Revue des Etudes Grecques 83 (1970) 333-350

Wohlers, Michael, Heilige Krankheit. Epilepsie in antiker Medizin, Astrologie und Religion (Marburg 1999)

112 Gesnerus 67 (2010) 